\title{
Nutritional Trends and Practices Among 6-23 Months Old Children: A Hospital-Based Cross- Sectional Study From Bhaktapur, Nepal
}

Sophiya Uprety ( $\sim$ sophiya@embracegoodnutrition.com )

Siddhi Memorial Hospital

\section{Anjita Khadka}

Siddhi Memorial Hospital

Alina Paudyal

Siddhi Memorial Hospital

Dhruba Shrestha

Siddhi Memorial Hospital

\section{Research Article}

Keywords: Breast feeding, IYCF, Infant and Young Child Feeding, child nutrition, urban nutrition, nutrition in transition, Nepal

Posted Date: December 31st, 2020

DOl: https://doi.org/10.21203/rs.3.rs-131657/v1

License: (c) (i) This work is licensed under a Creative Commons Attribution 4.0 International License. Read Full License 


\section{Abstract}

Background: Child undernutrition has been a prevailing issue in country like Nepal. Though there has been progress over the decades, the burden of child undernutrition still remains unacceptably high. It is important to look at how infants and young children's feeding practices and nutritional status are affected by nutrition transitioning in rapidly urbanizing Nepal

Methods: This is a cross-sectional research design Children aged 6-23 months and their mothers visiting the hospital for regular immunization services constituted the study population. Data was collected from 305 children over four months from September 2019 to January 2020. Anthropometric measurements (weight and length) were collected of all children and their mothers/caretakers were interviewed on the IYCF practices via a semi-structured questionnaire Data was analyzed using WHO Anthro Survey Analyzer, Microsoft Excel and STATA 15.

Results: Early initiation of breastfeeding was $47.9 \%$ and use of bottle was $48.5 \%$. Minimum dietary diversity, minimum meal frequency and minimum acceptable diet were $66.2 \%, 79.1 \%$ and $54.4 \%$ respectively. Consumption of nutrient-poor packaged foods was $63.0 \%$ with biscuits being the most common type (52.1\%) and $32.8 \%$ of the children were found to be drinking tea. Overall, $13.8 \%$ of the children were stunted, $3.9 \%$ were underweight and $1.7 \%$ were wasted while $2.3 \%$ were overweight.

Conclusion: The practices related to breastfeeding and diets of young children in urban areas of Nepal are not optimal with only around half meeting the minimum standards recommended by the WHO. Therefore, there is a pressing need to wake up to the rapidly changing dietary patterns among young children through necessary urban nutrition policies and programmatic interventions, the rising adverse nutrition issues can be nipped in the bud before they get too pervasive.

\section{Background}

Child undernutrition has been a prevailing issue in Nepal. Though there has been progress over the decades, the burden of child undernutrition still remains unacceptably high. Nepal Demographic and Health Survey (NDHS) 2016 reported that $36 \%$ of children under five years of age are stunted, $10 \%$ are wasted and $27 \%$ are underweight.(1) The same national survey presented disaggregation by residence which shows child undernutrition rates to be slightly better off in urban areas in comparison to rural areas, but are still high enough to be a significant public health concern. Stunting, underweight and wasting among children below five years residing in urban areas of Nepal are $32 \%, 23.4 \%$ and $9.2 \%$ respectively. (1) Of them, the prevalence of stunting (chronic malnutrition) is 'very high' as per the new child malnutrition thresholds by World Health Organization (WHO).(2)

'First 1000 days of life,' the period from conception till the child turns two years of age, is considered particularly crucial because most of the brain development takes place during this period. Nutrition plays a crucial role in intellectual, behavioral and physical development during this period.(3) Given the vulnerability of younger children, it is also important to take closer look into the malnutrition rates among 
the younger ages. Child undernutrition prevalence shown by NDHS 2016 among the overall younger age group of 6-23 months was $27.3 \%$ to be stunted, $26.7 \%$ to be underweight and $14.3 \%$ to be wasted. However, the disaggregation among the same younger age group is not available for urban areas.

Urbanization is a global phenomenon which is marked with an evident shift towards an unhealthy diet high in fat and processed foods and low in fiber, with corresponding increases in degenerative diseases. (4) Nepal is witnessing a rapid rate of urbanization. Though it is one of the least urbanized countries, it is also among the top 10 fastest urbanizing countries in the world.(5) This is an evolving area of importance for nutrition programming as well as research. Rising overweight and obesity due to 'nutrition in transition' is often a feature in urban areas of developing countries. $(6,7)$ This has already been indicated in Nepal among adults and adolescents. Overweight and obesity is significantly higher among urban men and women in comparison to their counterparts in rural areas: $26.1 \%$ versus $15.4 \%$ among women; and $17.1 \%$ versus $9.8 \%$ among men.(1) Similarly, Nepal Micronutrient Status Survey (NMNSS) 2016 documented the prevalence of overweight and obesity among adolescent boys and girls to be significantly higher in urban areas: $9.7 \%$ versus $3.9 \%$ among urban boys aged $10-19$ years and $9.9 \%$ versus $3.7 \%$ among urban adolescent girls aged 10-19 years.(8) Among all children less than five years of age, the prevalence of overweight and obesity is $1 \%$ (NDHS 2016) and the trend of higher overweight and obesity in urban areas among this age group is not yet reported by the national surveys. Delving further into the younger age group of 6-23 months, the overall overweight and obesity was found to be $1.4 \%$.(1)However, the disaggregation for urban areas is not available for children under two years of age.

Sub-optimal Infant and Young Child Feeding (IYCF) practices continue to remain as one of the key underlying determinants of child malnutrition with very slow progress made over the years. $(9,10)$ As per the NDHS 2016, less than half of all children aged 6-23 months, $47 \%$, receive the minimum dietary diversity and just above a third, $36 \%$, of the children aged 6-23 months receive a minimum acceptable diet in Nepal.

There is no significant difference between rural and urban areas in the standard IYCF indicators (MDD, MMF and MAD) among children aged 6-23 months as per the national surveys. (NDHS 2016, NMNSS 2016.) However, few researches focused in urban areas are unearthing other worrying dimensions that pose new challenges for IYCF and child nutritional status. For instance, Assessment in Child Feeding and Health $(\mathrm{ARCH})$ studies are documenting high unhealthy snack food consumptions with a recent one documenting that a quarter of calorie consumed by the young children was coming from unhealthy foods.(11)

It is thus now critical to continue the focus at how infants and young children's feeding behaviors and nutritional status are affected by the nutrition transitioning due to socio-economic and environmental changes in urban areas. We noticed a dearth of studies assessing both nutritional status as well as feeding practices (both the WHO recommended standard IYCF indicators and the unhealthy foods together) together among children aged 6-23 months in urban contexts of 
Nepal. Our study set out to fill this gap and hoped to shed more light on the extent of nutrition problem in urban/peri-urban areas of Kathmandu.

\section{Methods}

\section{Study population and data collection}

Siddhi Memorial Hospital (for Women and Children) located at Bhaktapur Nepal, an urban area, provided the setting for this study. The catchment areas of this hospital primarily include locals of Newar ethnicity who are long permanent residents of Bhaktapur as well as migrant residents from different parts of the country and residents of neighboring districts.

We adopted a cross-sectional research design with quantitative methodology. Healthy children aged 623 months and their mothers visiting the hospital for regular immunization services constituted the study population. Sample size estimations for this study were calculated based on the primary outcomes of interest for the overall study, which were to assess child malnutrition rates and child feeding practices with a confidence level of 95 percent and 5 percent margin of error. So, a sample size of 300 was deemed sufficient. Simple random sampling of the children was followed as there was no set pattern to select the children. Any random child visiting the hospital was selected until the sample size of at least 300 was reached. The inclusion criteria were children aged 6 months to 23 months along with their mother/caretaker residing in urban/peri- urban areas while children below 6 months and above 23 months of age, those with illnesses and not residing in urban/peri-urban area were excluded.

Data was collected from 305 children over four months from September 2019 to January 2020. Anthropometric measurements (weight and length) were collected of all children and their mothers/caretakers were interviewed on the IYCF practices via a semi-structured questionnaire. The study questionnaires were pretested among a different group of children and their mothers prior to the study. Children's ages were calculated from their date of birth and date of data collection. Data collection was randomly monitored by the study authors.

Outcomes of interest were children's nutritional status: the proportion of stunting, underweight wasting, overweight and obesity along with the corresponding mean z-scores; standard IYCF practices: proportion of children with continued breastfeeding, minimum dietary diversity, minimum meal frequency, minimum acceptable diet; plus frequency and types of nutrient-poor packaged foods consumption by the young children.

Ethical approval was received from Siddhi Memorial Hospital and Nepal Health Research Council (reference number 718-2019.) Also, information about the purpose of the study was verbally communicated to the mothers/caretakers to receive consent from them prior to data collection.

Data was analyzed using WHO Anthro Survey Analyzer, Microsoft Excel and STATA 15. 
All methods were carried out in accordance with relevant guidelines and regulations.

\section{Results}

Demographic and socio-economic characteristics of children and their caregivers are presented in Table 1. Slightly more males (53.1\%) than females (46.9\%) were sampled and as expected, there were more children in the older age group of 12-23 months (58.4\%) than among 6-11 months (41.6\%.) As reported by mothers/caretakers, most of the children (88\%), had normal birth weight, i.e., more than 2.5 kilograms. Almost four out of 10 (37.4\%) were born through cesarean section delivery. Given the location of the hospital amidst a traditional Newar community, more than half of the sampled children were of Newar ethnicity (55\%), followed by Janjati (26.9\%) and Chhetri (11.1\%). (Table 1) 
Table 1

Demographic and socio-economic

characteristics of sampled children aged 6-23

months

\begin{tabular}{|lll|}
\hline Characteristics & N & $\%$ \\
\hline Gender & & \\
\hline Female & 143 & 46.9 \\
\hline Male & 162 & 53.1 \\
\hline Age in months & & \\
\hline 11-Jun & 127 & 41.6 \\
\hline 23-Dec & 178 & 58.4 \\
\hline Birth weight & & \\
\hline Low birth weight $(<2.5 \mathrm{~kg})$ & 30 & 9.8 \\
\hline Normal birth weight $(>2.5 \mathrm{~kg})$ & 270 & 88.5 \\
\hline NA & 5 & 1.6 \\
\hline Type of delivery/birth & & \\
\hline Vaginal & 191 & 62.6 \\
\hline Cesarean Section & 114 & 37.4 \\
\hline Ethnicity & & \\
\hline Brahmin & 15 & 4.9 \\
\hline Chhetri & 34 & 11.1 \\
\hline Dalit & 6 & 2 \\
\hline Janajati & 85.9 \\
\hline Newar & & \\
\hline
\end{tabular}

The majority of primary caregivers were mothers of the children (79.3\%) followed by grandmothers $(11.5 \%)$ while fathers were the primary caregivers of less than two percent of the children. There were very few mothers who had never attended school (4.3\%) while most of the mothers had attended six to 10 years of school, i.e., secondary level education (33.8\%) followed by 11 to 12 years of school, i.e., higher secondary level (26.6\%) and Bachelor's degree (20\%) but very few had a Master's degree $(6.2 \%)$. Fathers' education levels was also similar to that of the mothers. More than two third of the mothers were homemakers $(67.2 \%)$ while around a third (32.5\%) were engaged in some form of income generating activities. (Table 2) 
Table 2

Characteristics of caregivers of the enrolled children

\begin{tabular}{|lll|}
\hline Characteristics & N & $\%$ \\
\hline Primary caretaker & & \\
\hline Mother & 242 & 79.3 \\
\hline Father & 5 & 1.6 \\
\hline Grandmother & 35 & 11.5 \\
\hline Mother \& Father & 8 & 2.6 \\
\hline Mother \& Grandmother & 4 & 1.3 \\
\hline Father \& Grandmother & 3 & 1 \\
\hline Others & 8 & 2.6 \\
\hline Mother's education & & \\
\hline No education (never attended school) & 13 & 4.3 \\
\hline Primary (0-5 years of school) & 28 & 9.2 \\
\hline Secondary (6-10 years of school) & 103 & 33.8 \\
\hline Higher secondary (11-12 years of school) & 81 & 26.6 \\
\hline Bachelor's degree & 61 & 20 \\
\hline Master's degree & 19 & 6.2 \\
\hline Father's education & 205 & 67.2 \\
\hline No education (never attended school) & 10 & 3.3 \\
\hline Primary (0-5 years of school) & 33 & 10.8 \\
\hline Secondary (6-10 years of school) & 102 & 33.4 \\
\hline Higher secondary (11-12 years of school) & 78 & 25.6 \\
\hline Bachelor's degree & 59 & 19.3 \\
\hline Master's degree & 19 & 6.2 \\
\hline NA & 4 & 1.3 \\
\hline Mother's occupation & & \\
\hline Homemaker & & \\
\hline Engaged in income generation & & \\
\hline NA & & \\
\hline
\end{tabular}




\begin{tabular}{|lll|}
\hline Characteristics & N & $\%$ \\
\hline Father's occupation & & \\
\hline Foreign employment & 19 & 6.2 \\
\hline Other profession & 282 & 92.5 \\
\hline NA & 4 & 1.3 \\
\hline
\end{tabular}

Infant and Young Child Feeding practices

Table 3 provides information on infant and young child feeding practices. Breastfeeding was nearly universal amongst the sampled children as almost all of them (97.7\%) were breastfed at some time. Breastfeeding rates were still almost equally high (94.1\%) at one year of age. However, only less than half of the children (47.9\%) were breastfed within one hour of birth. Almost half $(48.5 \%)$ of the children were fed with a bottle in the previous 24 hours.

Looking at the percentage of children aged 6-23 months who were fed according to the minimum recommended standards of the WHO: overall, two-thirds (66.2\%) of the children had received the minimum dietary diversity, almost eight out of ten $(79.1 \%)$ had received the minimum meal frequency and little more than half $(54.4 \%)$ had met the criteria of minimum acceptable diet the previous day.

Table 3

Infant and young child feeding practices of sampled children aged 6-23 months as provided by their mothers/caretakers

\begin{tabular}{|llll|}
\hline IYCF practices & Total & $\boldsymbol{n}$ & $\%$ \\
\hline Children who were ever breastfed & 305 & 298 & 97.7 \\
\hline Children with timely initiation of breastfeeding (within 1 hour) & 305 & 146 & 47.9 \\
\hline Children 12-15 months with continued breastfeeding at 1 year & 119 & 112 & 94.1 \\
\hline Children 20-23 months with continued breastfeeding at 2 years & 4 & 4 & 100 \\
\hline Children who were bottle fed & 305 & 148 & 48.5 \\
\hline Children 6-8 months with timely initiation of complementary foods & 4 & 4 & 100 \\
\hline Children who received the minimum dietary diversity & 305 & 202 & 66.2 \\
\hline Children who received the minimum meal frequency & 287 & 227 & 79.1 \\
\hline Children who received the minimum acceptable diet & 287 & 156 & 54.4 \\
\hline Mean number of food groups fed & 4 & & \\
\hline
\end{tabular}

Note: 
1 Minimum dietary diversity: Intake from at least four of the seven main food groups in the previous day. (The seven food groups include grains, roots and tubers, legumes and nuts, dairy products (milk, yogurt, and cheese), flesh foods (meat, fish, poultry and liver/organ meats), eggs, vitamin A rich fruits and vegetables, and other fruits and vegetables.)

2Minimum meal frequency: The child consuming the minimum number of solid, semi-solid or soft food snacks/meals the previous day. (two or more times per day for a breastfed child aged 6-8 months; three or more times for a breastfed child aged 9-23 months and four or more times for non-breastfed children aged 6-23 months. Meals include both meals and snacks)

3Minimum acceptable diet: The composite of the minimum meal frequency and minimum dietary diversity consumed by the children currently breastfeeding in the previous day. (For non-breastfeeding children, it is the composite of children who had at least 2 milk feeds and had the minimum dietary not including milk feeds, and the minimum meal frequency the previous day.)

Consumption of nutrient-poor packaged foods

Table 4 presents the practices related to the consumption of unhealthy packaged foods by the children aged 6-23 months.

Almost two third (63.0\%) of the children aged 6-23 months had consumed nutrient-poor packaged foods in the past 24 hours. Similar proportion of them $(62.7 \%)$ had consumed at least once while a quarter of them $(25.4 \%)$ had consumed twice. Majority of the children were given those foods by mothers $(64.1 \%)$ followed by fathers (12.5\%) and grandmothers (9.4\%). Biscuits was the most commonly given packaged food $(52.1 \%)$ followed by chocolates $(17.7 \%)$ and to a lesser extents various combinations of the nutrientpoor packaged foods. In addition, one third of the children (32.8\%) were given tea in the previous 24 hours and mothers mentioned that often it was together with the biscuits. 
Table 4

Practices of nutrient-poor packaged foods consumption of sampled children aged 6-23 months as provided by their mothers/caretaker

\begin{tabular}{|c|c|c|}
\hline Consumption of nutrient-poor packaged foods & $N$ & $\%$ \\
\hline \multicolumn{3}{|l|}{ Intake of any sugary or salty packaged food } \\
\hline Yes & 192 & 63 \\
\hline No & 112 & 36.7 \\
\hline Don't know & 1 & 0.3 \\
\hline \multicolumn{3}{|c|}{ If yes, then frequency of intake of any sugary or salty packaged food } \\
\hline 1 & 121 & 62.7 \\
\hline 2 & 49 & 25.4 \\
\hline 3 & 14 & 7.3 \\
\hline$>3$ & 8 & 4.1 \\
\hline \multicolumn{3}{|c|}{ Who usually gives the sugary or salty packaged food } \\
\hline Mother & 123 & 64.1 \\
\hline Father & 24 & 12.5 \\
\hline Grandmother & 18 & 9.4 \\
\hline Grandfather & 3 & 1.6 \\
\hline Mother \& Father & 5 & 2.6 \\
\hline Siblings & 12 & 6.3 \\
\hline Other relatives & 7 & 3.6 \\
\hline \multicolumn{3}{|l|}{ Type of sugary or salty packaged food given } \\
\hline Chocolates & 34 & 17.7 \\
\hline Biscuits & 100 & 52.1 \\
\hline Chocolates and biscuits & 17 & 8.9 \\
\hline Chips or Cheeseball & 15 & 7.8 \\
\hline Noodles & 6 & 3.1 \\
\hline Pastries & 1 & 0.5 \\
\hline biscuits \& cheeseball & 2 & 1 \\
\hline Biscuits \& Noodles & 4 & 2.1 \\
\hline
\end{tabular}




\begin{tabular}{|lll|}
\hline Consumption of nutrient-poor packaged foods & N & $\%$ \\
\hline biscuits \& pastries & 5 & 2.6 \\
\hline Chocolates \& cheeseball & 1 & 0.5 \\
\hline chocolates \& noodles & 1 & 0.5 \\
\hline Chocolates \& pastries & 1 & 0.5 \\
\hline Noodles \& Cheeseball & 1 & 0.5 \\
\hline noodles \& pastries & 1 & 0.5 \\
\hline chocolates, biscuits \& pastries & 1 & 0.5 \\
\hline chocolates, biscuits \& noodles & 1 & 0.5 \\
\hline biscuits, pastries \& noodles & 1 & 0.5 \\
\hline
\end{tabular}

Child malnutrition status

The prevalence of stunting, underweight, wasting and overweight/obesity among the sampled children aged 6-23 months are presented in Table 5. Overall, $13.8 \%$ of the children were found to be stunted, $3.9 \%$ to be underweight and $1.7 \%$ to be wasted while $2.3 \%$ were overweight. Disaggregation for these indicators were conducted by age, sex, maternal and paternal education levels, ethnicity, birth weight status and delivery types.

Undernutrition was slightly higher amongst males than in females in all three indices. Stunting prevalence with $95 \%$ confidence interval was $14.8 \%(10.1 ; 21.2)$ among males versus $12.7 \%(8.1 ; 19.3)$ among females; underweight prevalence with $95 \%$ confidence interval was $4.9 \%(2.5 ; 9.6)$ among males versus $2.8 \%(1.0 ; 7.3)$ among females; and wasting prevalence with $95 \%$ confidence interval was $1.9 \%$ $(0.6 ; 5.7)$ among males versus $1.4 \%(0.3 ; 5.5)$ among females. Overweight and obesity on the other hand was found to be slightly higher among females with its prevalence with $95 \%$ confidence interval being $2.8 \%(1.1 ; 7.3)$ among females versus $1.9 \%(0.6 ; 5.7)$ among males.

Stunting was higher in older age groups among the 12-23 month olds in comparison to 6-11 month olds $17.1 \%(12.2 ; 23.5)$ versus $9.3 \%(5.3 ; 15.7)$ respectively.

Stunting and underweight prevalence were higher among children who were born with low birth weight in comparison to born with normal birth weight: stunting prevalence with $95 \%$ confidence interval was $23.3 \%(11.5 ; 41.6)$ among those who were reported to be born with low birth weight versus $13.0 \%(9.5$; 17.6) among those reported to be born with normal birth weight; underweight prevalence with $95 \%$ confidence interval was $6.7 \%(1.7 ; 23.2)$ among children with low birth weight versus $3.7 \%(2.0 ; 6.8)$ among those who had normal birth weights. However, wasting as well as overweight and obesity was found only among those born normal birth weight in this sample but was not present among children born low birth weight. Amongst children who were born normal birth weight, the wasting prevalence with 
$95 \%$ confidence interval was $2.6 \%(1.2 ; 5.4)$ and overweight/obesity prevalence with $95 \%$ confidence interval was $1.1 \%(0.4 ; 3.4)$.

The findings did not show any consistent pattern in the malnutrition status of the children by parental education levels and by the type of delivery. With regards to child's ethnicity, Chhetri children had the highest levels of stunting and underweight as well as overweight/obesity but not wasting which was present only among Newar children (table 5).

Table 5: Stunting, underweight, wasting and overweight/obesity among sampled children aged 6-23 months and disaggregation by selected characteristics

Height-for-Age 


\begin{tabular}{|c|c|c|c|c|c|c|}
\hline Group & $\underset{N}{\text { Weighted }}$ & $\begin{array}{l}\text { Unweighted } \\
N\end{array}$ & $\begin{array}{l}-3 S D(95 \% \\
C l)\end{array}$ & $\begin{array}{l}-2 S D(95 \% \\
C I)\end{array}$ & $\begin{array}{l}\text { z-score mean } \\
(95 \% \mathrm{Cl})\end{array}$ & $\begin{array}{l}Z \text {-score } \\
\text { SD }\end{array}$ \\
\hline All children & 304 & 304 & $\begin{array}{l}2.0(0.9 ; \\
4.3)\end{array}$ & $\begin{array}{l}13.8(10.4 ; \\
18.2)\end{array}$ & $-0.7(-0.9 ;-0.6)$ & 1.14 \\
\hline \multicolumn{7}{|c|}{ Child's age group: } \\
\hline 06-11 mo & 129 & 129 & $\begin{array}{l}1.6(0.4 ; \\
6.0)\end{array}$ & $\begin{array}{l}9.3(5.3 ; \\
15.7)\end{array}$ & $-0.5(-0.7 ;-0.3)$ & 1.07 \\
\hline $12-23 \mathrm{mo}$ & 175 & 175 & $\begin{array}{l}2.3(0.9 ; \\
6.0)\end{array}$ & $\begin{array}{l}17.1(12.2 ; \\
23.5)\end{array}$ & $-0.9(-1.0 ;-0.7)$ & 1.18 \\
\hline \multicolumn{7}{|l|}{ Child's Sex: } \\
\hline Female & 142 & 142 & $\begin{array}{l}1.4(0.3 ; \\
5.5)\end{array}$ & $\begin{array}{l}\text { 12.7 (8.1; } \\
\text { 19.3) }\end{array}$ & $-0.6(-0.8 ;-0.5)$ & 1.13 \\
\hline Male & 162 & 162 & $\begin{array}{l}2.5(0.9 ; \\
6.4)\end{array}$ & $\begin{array}{l}14.8(10.1 ; \\
21.2)\end{array}$ & $-0.8(-1.0 ;-0.6)$ & 1.15 \\
\hline \multicolumn{7}{|c|}{ Maternal Education: } \\
\hline No Education & 13 & 13 & $\begin{array}{l}0.0(0.0 \\
0.0)\end{array}$ & $\begin{array}{l}7.7(1.1 \\
39.3)\end{array}$ & $-1.0(-1.4 ;-0.7)$ & 0.66 \\
\hline Primary & 28 & 28 & $\begin{array}{l}0.0(0.0 ; \\
0.0)\end{array}$ & $\begin{array}{l}10.7(3.5 ; \\
28.6)\end{array}$ & $-0.7(-1.1 ;-0.3)$ & 1.04 \\
\hline Secondary & 102 & 102 & $\begin{array}{l}1.9(0.5 \\
7.5)\end{array}$ & $\begin{array}{l}20.4(13.7 ; \\
29.3)\end{array}$ & $-0.9(-1.1 ;-0.7)$ & 1.06 \\
\hline $\begin{array}{l}\text { Higher } \\
\text { Secondary }\end{array}$ & 81 & 81 & $\begin{array}{l}1.2(0.2 ; \\
8.3)\end{array}$ & $\begin{array}{l}6.2(2.6 \\
14.0)\end{array}$ & $-0.6(-0.8 ;-0.4)$ & 1 \\
\hline Bachelors & 60 & 60 & $\begin{array}{l}3.3(0.8 \\
12.5)\end{array}$ & $\begin{array}{l}15.0(8.0 ; \\
26.5)\end{array}$ & $-0.6(-1.0 ;-0.3)$ & 1.36 \\
\hline Masters & 19 & 19 & $\begin{array}{l}5.3(0.7 \\
29.6)\end{array}$ & $\begin{array}{l}15.8(5.1 \\
39.3)\end{array}$ & $-0.4(-1.1 ; 0.3)$ & 1.61 \\
\hline \multicolumn{7}{|c|}{ Father's Education: } \\
\hline No Education & 10 & 10 & $\begin{array}{l}0.0(0.0 ; \\
0.0)\end{array}$ & $\begin{array}{l}10.0(1.4 \\
47.0)\end{array}$ & $-0.6(-1.3 ; 0.1)$ & 1.2 \\
\hline Primary & 33 & 33 & $\begin{array}{l}0.0(0.0 \\
0.0)\end{array}$ & $\begin{array}{l}12.1 \\
28.3)\end{array}$ & $-0.8(-1.1 ;-0.5)$ & 0.91 \\
\hline Secondary & 102 & 102 & $\begin{array}{l}2.0(0.5 \\
7.6)\end{array}$ & $\begin{array}{l}12.7(7.5 \\
20.8)\end{array}$ & $-0.8(-1.0 ;-0.6)$ & 1.06 \\
\hline $\begin{array}{l}\text { Higher } \\
\text { Secondary }\end{array}$ & 78 & 78 & $\begin{array}{l}3.8(1.2 \\
11.3)\end{array}$ & $\begin{array}{l}16.7(9.9 ; \\
26.7)\end{array}$ & $-0.9(-1.2 ;-0.6)$ & 1.3 \\
\hline Bachelors & 58 & 58 & $\begin{array}{l}1.7(0.2 ; \\
11.4)\end{array}$ & $\begin{array}{l}\text { 13.8 ( } 7.0 \\
25.3)\end{array}$ & $-0.5(-0.8 ;-0.2)$ & 1.16 \\
\hline
\end{tabular}




\begin{tabular}{|c|c|c|c|c|c|c|}
\hline Group & $\begin{array}{l}\text { Weighted } \\
N\end{array}$ & $\begin{array}{l}\text { Unweighted } \\
N\end{array}$ & $\begin{array}{l}-3 S D(95 \% \\
C I)\end{array}$ & $\begin{array}{l}-2 S D(95 \% \\
C I)\end{array}$ & $\begin{array}{l}\text { z-score mean } \\
(95 \% \mathrm{Cl})\end{array}$ & $\begin{array}{l}\text { Z-score } \\
S D\end{array}$ \\
\hline Masters & 19 & 19 & $\begin{array}{l}0.0(0.0 ; \\
0.0)\end{array}$ & $\begin{array}{l}10.5(2.6 ; \\
33.9)\end{array}$ & $-0.2(-0.7 ; 0.3)$ & 1.1 \\
\hline \multicolumn{7}{|c|}{ Child's Ethnicity: } \\
\hline Brahmin & 15 & 15 & $\begin{array}{l}6.7(0.9 \\
35.5)\end{array}$ & $\begin{array}{l}13.3(3.3 ; \\
40.7)\end{array}$ & $-0.6(-1.2 ; 0.1)$ & 1.37 \\
\hline Chhetri & 34 & 34 & $\begin{array}{l}2.9(0.4 \\
18.3)\end{array}$ & $\begin{array}{l}23.5(12.2 ; \\
40.6)\end{array}$ & $-0.9(-1.3 ;-0.5)$ & 1.23 \\
\hline Dalit & 6 & 6 & $\begin{array}{l}0.0(0.0 ; \\
0.0)\end{array}$ & $\begin{array}{l}\text { 16.7 ( 2.3; } \\
63.4)\end{array}$ & $-0.9(-1.5 ;-0.4)$ & 0.77 \\
\hline Janajati & 82 & 82 & $\begin{array}{l}1.2(0.2 \\
8.2)\end{array}$ & $\begin{array}{l}9.8(4.9 \\
18.4)\end{array}$ & $-0.8(-1.0 ;-0.5)$ & 1.01 \\
\hline Newar & 167 & 167 & $\begin{array}{l}1.8(0.6 \\
5.5)\end{array}$ & $\begin{array}{l}13.8(9.3 ; \\
19.9)\end{array}$ & $-0.7(-0.9 ;-0.5)$ & 1.18 \\
\hline \multicolumn{7}{|c|}{ Birthweight Status } \\
\hline LBW & 30 & 30 & $\begin{array}{l}6.7(1.7 \\
23.2)\end{array}$ & $\begin{array}{l}23.3(11.5 ; \\
41.6)\end{array}$ & $-1.2(-1.5 ;-0.8)$ & 1.01 \\
\hline NBW & 269 & 269 & $\begin{array}{l}1.5(0.6 \\
3.9)\end{array}$ & $\begin{array}{l}13.0(9.5 ; \\
17.6)\end{array}$ & $-0.7(-0.8 ;-0.5)$ & 1.15 \\
\hline \multicolumn{7}{|c|}{ Delivery Type } \\
\hline $\begin{array}{l}\text { Cesarean } \\
\text { Section }\end{array}$ & 113 & 113 & $\begin{array}{l}2.7(0.9 ; \\
8.0)\end{array}$ & $\begin{array}{l}12.4(7.5 ; \\
19.9)\end{array}$ & $-0.6(-0.8 ;-0.4)$ & 1.08 \\
\hline Vaginal & 191 & 191 & $\begin{array}{l}1.6(0.5 \\
4.8)\end{array}$ & $\begin{array}{l}\text { 14.7 (10.3; } \\
20.5)\end{array}$ & $-0.8(-0.9 ;-0.6)$ & 1.18 \\
\hline
\end{tabular}

Weight-for-Age 


\begin{tabular}{|c|c|c|c|c|c|c|}
\hline \multirow[t]{2}{*}{ Group } & \multirow{2}{*}{$\begin{array}{l}\text { Weighted } \\
N\end{array}$} & \multirow{2}{*}{$\begin{array}{l}\text { Unweighted } \\
N\end{array}$} & \multirow{2}{*}{$\begin{array}{l}-3 S D(95 \% \\
C I)\end{array}$} & \multirow{2}{*}{$\begin{array}{l}-2 S D(95 \% \\
C I)\end{array}$} & \multirow{2}{*}{$\begin{array}{l}\text { z-score mean } \\
(95 \% \mathrm{Cl})\end{array}$} & \multirow[b]{2}{*}{$\begin{array}{l}\text { Z-score } \\
\text { SD }\end{array}$} \\
\hline & & & & & & \\
\hline All & 305 & 305 & $\begin{array}{l}0.3(0.0 ; \\
2.3)\end{array}$ & $\begin{array}{l}3.9(2.2 ; \\
6.8)\end{array}$ & $-0.3(-0.4 ;-0.2)$ & 1.08 \\
\hline \multicolumn{7}{|c|}{ Child's age group: } \\
\hline 06-11 mo & 129 & 129 & $\begin{array}{l}0.0(0.0 ; \\
0.0)\end{array}$ & $\begin{array}{l}3.9(1.6 ; \\
9.0)\end{array}$ & $-0.3(-0.5 ;-0.1)$ & 1.16 \\
\hline $12-23 \mathrm{mo}$ & 176 & 176 & $\begin{array}{l}0.6(0.1 ; \\
4.0)\end{array}$ & $\begin{array}{l}4.0(1.9 ; \\
8.1)\end{array}$ & $-0.3(-0.5 ;-0.2)$ & 1.01 \\
\hline \multicolumn{7}{|l|}{ Child's Sex: } \\
\hline Female & 143 & 143 & $\begin{array}{l}0.0(0.0 ; \\
0.0)\end{array}$ & $\begin{array}{l}2.8(1.0 \\
7.3)\end{array}$ & $-0.3(-0.5 ;-0.1)$ & 1.05 \\
\hline Male & 162 & 162 & $\begin{array}{l}0.6(0.1 ; \\
4.3)\end{array}$ & $\begin{array}{l}4.9(2.5 ; \\
9.6)\end{array}$ & $-0.3(-0.5 ;-0.2)$ & 1.1 \\
\hline \multicolumn{7}{|c|}{ Maternal Education: } \\
\hline No Education & 13 & 13 & $\begin{array}{l}1.6(0.2 \\
10.8)\end{array}$ & $\begin{array}{l}4.9(1.6 \\
14.2)\end{array}$ & $-0.1(-0.4 ; 0.2)$ & 1.17 \\
\hline Primary & 28 & 28 & $\begin{array}{l}0.0(0.0 \\
0.0)\end{array}$ & $\begin{array}{l}0.0(0.0 \\
0.0)\end{array}$ & $-0.3(-0.4 ;-0.1)$ & 0.81 \\
\hline Secondary & 102 & 102 & $\begin{array}{l}0.0(0.0 ; \\
0.0)\end{array}$ & $\begin{array}{l}0.0(0.0 ; \\
0.0)\end{array}$ & $0.1(-0.5 ; 0.7)$ & 1.38 \\
\hline $\begin{array}{l}\text { Higher } \\
\text { Secondary }\end{array}$ & 81 & 81 & $\begin{array}{l}0.0(0.0 ; \\
0.0)\end{array}$ & $\begin{array}{l}7.7(1.1 ; \\
39.3)\end{array}$ & $-0.8(-1.3 ;-0.4)$ & 0.83 \\
\hline Bachelors & 60 & 60 & $\begin{array}{l}0.0(0.0 \\
0.0)\end{array}$ & $\begin{array}{l}3.6(0.5 \\
21.6)\end{array}$ & $-0.4(-0.8 ; 0.0)$ & 1.11 \\
\hline Masters & 19 & 19 & $\begin{array}{l}0.0(0.0 ; \\
0.0)\end{array}$ & $\begin{array}{l}6.8(3.3 ; \\
13.6)\end{array}$ & $-0.5(-0.7 ;-0.3)$ & 1.13 \\
\hline \multicolumn{7}{|c|}{ Father's Education: } \\
\hline No Education & 59 & 59 & $\begin{array}{l}0.0(0.0 \\
0.0)\end{array}$ & $\begin{array}{l}3.4(0.8 \\
12.7)\end{array}$ & $0.0(-0.3 ; 0.3)$ & 1.14 \\
\hline Primary & 78 & 78 & $\begin{array}{l}1.3(0.2 ; \\
8.6)\end{array}$ & $\begin{array}{l}2.6(0.6 ; \\
9.7)\end{array}$ & $-0.3(-0.6 ;-0.1)$ & 1.05 \\
\hline Secondary & 19 & 19 & $\begin{array}{l}0.0(0.0 \\
0.0)\end{array}$ & $\begin{array}{l}0.0(0.0 \\
0.0)\end{array}$ & $0.1(-0.2 ; 0.5)$ & 0.74 \\
\hline $\begin{array}{l}\text { Higher } \\
\text { Secondary }\end{array}$ & 10 & 10 & $\begin{array}{l}0.0(0.0 ; \\
0.0)\end{array}$ & $\begin{array}{l}0.0(0.0 ; \\
0.0)\end{array}$ & $0.0(-1.1 ; 1.0)$ & 1.82 \\
\hline
\end{tabular}




\begin{tabular}{|c|c|c|c|c|c|c|}
\hline \multirow[t]{2}{*}{ Group } & \multirow{2}{*}{$\begin{array}{l}\text { Weighted } \\
N\end{array}$} & \multirow{2}{*}{ Unweighted } & \multirow{2}{*}{$\begin{array}{l}-3 S D(95 \% \\
C I)\end{array}$} & \multirow{2}{*}{$\begin{array}{l}-2 S D(95 \% \\
C I)\end{array}$} & \multirow{2}{*}{$\begin{array}{l}\text { z-score mean } \\
(95 \% \mathrm{Cl})\end{array}$} & \multirow[b]{2}{*}{$\begin{array}{l}\text { z-score } \\
\text { SD }\end{array}$} \\
\hline & & & & & & \\
\hline Bachelors & 33 & 33 & $\begin{array}{l}0.0(0.0 ; \\
0.0)\end{array}$ & $\begin{array}{l}9.1(2.9 ; \\
24.8)\end{array}$ & $-0.5(-0.8 ;-0.2)$ & 0.94 \\
\hline Masters & 102 & 102 & $\begin{array}{l}0.0(0.0 ; \\
0.0)\end{array}$ & $\begin{array}{l}4.9(2.0 ; \\
11.3)\end{array}$ & $-0.5(-0.7 ;-0.4)$ & 1 \\
\hline \multicolumn{7}{|c|}{ Child's Ethnicity: } \\
\hline Brahmin & 15 & 15 & $\begin{array}{l}0.0(0.0 ; \\
0.0)\end{array}$ & $\begin{array}{l}0.0(0.0 \\
0.0)\end{array}$ & $-0.4(-0.7 ; 0.0)$ & 0.72 \\
\hline Chhetri & 34 & 34 & $\begin{array}{l}0.0(0.0 ; \\
0.0)\end{array}$ & $\begin{array}{l}5.9(1.5 \\
20.8)\end{array}$ & $-0.4(-0.8 ;-0.1)$ & 1.08 \\
\hline Dalit & 6 & 6 & $\begin{array}{l}0.0(0.0 ; \\
0.0)\end{array}$ & $\begin{array}{l}0.0(0.0 ; \\
0.0)\end{array}$ & $-0.9(-1.4 ;-0.3)$ & 0.75 \\
\hline Janajati & 82 & 82 & $\begin{array}{l}0.0(0.0 ; \\
0.0)\end{array}$ & $\begin{array}{l}4.9(1.8 \\
12.3)\end{array}$ & $-0.4(-0.6 ;-0.2)$ & 0.93 \\
\hline Newar & 168 & 168 & $\begin{array}{l}0.6(0.1 ; \\
4.1)\end{array}$ & $\begin{array}{l}3.6(1.6 \\
7.8)\end{array}$ & $-0.2(-0.4 ; 0.0)$ & 1.17 \\
\hline \multicolumn{7}{|c|}{ Birthweight Status } \\
\hline LBW & 30 & 30 & $\begin{array}{l}0.0(0.0 ; \\
0.0)\end{array}$ & $\begin{array}{l}6.7(1.7 ; \\
23.2)\end{array}$ & $-0.8(-1.1 ;-0.5)$ & 0.8 \\
\hline NBW & 270 & 270 & $\begin{array}{l}0.4(0.1 ; \\
2.6)\end{array}$ & $\begin{array}{l}3.7(2.0 ; \\
6.8)\end{array}$ & $-0.3(-0.4 ;-0.1)$ & 1.09 \\
\hline \multicolumn{7}{|c|}{ Delivery Type } \\
\hline $\begin{array}{l}\text { Cesarean } \\
\text { Section }\end{array}$ & 114 & 114 & $\begin{array}{l}0.0(0.0 ; \\
0.0)\end{array}$ & $\begin{array}{l}2.6(0.8 \\
7.9)\end{array}$ & $-0.1(-0.3 ; 0.0)$ & 0.96 \\
\hline Vaginal & 191 & 191 & $\begin{array}{l}0.5(0.1 ; \\
3.7)\end{array}$ & $\begin{array}{l}4.7(2.5 ; \\
8.8)\end{array}$ & $-0.4(-0.6 ;-0.3)$ & 1.13 \\
\hline
\end{tabular}

Weight-for-Height 


\begin{tabular}{|c|c|c|c|c|c|c|c|c|}
\hline Group & $\begin{array}{l}\text { Weighted } \\
\text { N }\end{array}$ & $\begin{array}{l}\text { Unweighted } \\
N\end{array}$ & $\begin{array}{l}-3 S D \\
(95 \% \\
C I)\end{array}$ & $\begin{array}{l}-2 S D \\
(95 \% \\
C I)\end{array}$ & $\begin{array}{l}+2 S D \\
(95 \% \\
C I)\end{array}$ & $\begin{array}{l}+3 S D \\
(95 \% \\
C I)\end{array}$ & $\begin{array}{l}\text { z-score } \\
\text { mean } \\
(95 \% \mathrm{Cl})\end{array}$ & $\begin{array}{l}z- \\
\text { score } \\
S D\end{array}$ \\
\hline All & 303 & 303 & $\begin{array}{l}0.0 \\
(0.0 \\
0.0)\end{array}$ & $\begin{array}{l}1.7 \\
(0.7 \\
3.9)\end{array}$ & $\begin{array}{l}2.3 \\
(1.1 ; \\
4.8)\end{array}$ & $\begin{array}{l}1.0 \\
(0.3 ; \\
3.0)\end{array}$ & $\begin{array}{l}0.0(-0.1 \\
0.2)\end{array}$ & 1.05 \\
\hline \multicolumn{9}{|c|}{ Child's age group: } \\
\hline $06-11 \mathrm{mo}$ & 128 & 128 & $\begin{array}{l}0.0 \\
(0.0 \\
0.0)\end{array}$ & $\begin{array}{l}1.6 \\
(0.4 \\
6.1)\end{array}$ & $\begin{array}{l}3.1 \\
(1.2 ; \\
8.1)\end{array}$ & $\begin{array}{l}2.3 \\
(0.8 \\
7.1)\end{array}$ & $\begin{array}{l}-0.1(-0.3 \\
0.1)\end{array}$ & 1.1 \\
\hline $12-23 \mathrm{mo}$ & 175 & 175 & $\begin{array}{l}0.0 \\
(0.0 \\
0.0)\end{array}$ & $\begin{array}{l}1.7 \\
(0.6 \\
5.2)\end{array}$ & $\begin{array}{l}1.7 \\
(0.6 \\
5.2)\end{array}$ & $\begin{array}{l}0.0 \\
(0.0 \\
0.0)\end{array}$ & $\begin{array}{l}0.1(0.0 \\
0.3)\end{array}$ & 1 \\
\hline \multicolumn{9}{|l|}{ Child's Sex: } \\
\hline Female & 142 & 142 & $\begin{array}{l}0.0 \\
(0.0 \\
0.0)\end{array}$ & $\begin{array}{l}1.4 \\
(0.3 \\
5.5)\end{array}$ & $\begin{array}{l}2.8 \\
(1.1 ; \\
7.3)\end{array}$ & $\begin{array}{l}1.4 \\
(0.3 \\
5.5)\end{array}$ & $\begin{array}{l}0.0(-0.1 \\
0.2)\end{array}$ & 1.05 \\
\hline Male & 161 & 161 & $\begin{array}{l}0.0 \\
(0.0 \\
0.0)\end{array}$ & $\begin{array}{l}1.9 \\
(0.6 \\
5.7)\end{array}$ & $\begin{array}{l}1.9 \\
(0.6 \\
5.7)\end{array}$ & $\begin{array}{l}0.6 \\
(0.1 ; \\
4.3)\end{array}$ & $\begin{array}{l}0.0(-0.1 \\
0.2)\end{array}$ & 1.05 \\
\hline \multicolumn{9}{|c|}{ Maternal Education: } \\
\hline $\begin{array}{l}\text { No } \\
\text { Education }\end{array}$ & 13 & 13 & $\begin{array}{l}0.0 \\
(0.0 \\
0.0)\end{array}$ & $\begin{array}{l}1.7 \\
(0.2 ; \\
11.0)\end{array}$ & $\begin{array}{l}1.7 \\
(0.2 ; \\
11.0)\end{array}$ & $\begin{array}{l}0.0 \\
(0.0 ; \\
0.0)\end{array}$ & $\begin{array}{l}0.3(0.0 \\
0.5)\end{array}$ & 1.12 \\
\hline Primary & 28 & 28 & $\begin{array}{l}0.0 \\
(0.0 \\
0.0)\end{array}$ & $\begin{array}{l}1.2 \\
(0.2 \\
8.3)\end{array}$ & $\begin{array}{l}0.0 \\
(0.0 \\
0.0)\end{array}$ & $\begin{array}{l}0.0 \\
(0.0 \\
0.0)\end{array}$ & $\begin{array}{l}0.0(-0.2 \\
0.2)\end{array}$ & 0.91 \\
\hline Secondary & 102 & 102 & $\begin{array}{l}0.0 \\
(0.0 \\
0.0)\end{array}$ & $\begin{array}{l}0.0 \\
(0.0 \\
0.0)\end{array}$ & $\begin{array}{l}5.3 \\
(0.7 ; \\
29.6)\end{array}$ & $\begin{array}{l}5.3 \\
(0.7 ; \\
29.6)\end{array}$ & $\begin{array}{l}0.4(-0.1 \\
0.9)\end{array}$ & 1.18 \\
\hline $\begin{array}{l}\text { Higher } \\
\text { Secondary }\end{array}$ & 81 & 81 & $\begin{array}{l}0.0 \\
(0.0 \\
0.0)\end{array}$ & $\begin{array}{l}0.0 \\
(0.0 \\
0.0)\end{array}$ & $\begin{array}{l}0.0 \\
(0.0 \\
0.0)\end{array}$ & $\begin{array}{l}0.0 \\
(0.0 \\
0.0)\end{array}$ & $\begin{array}{l}-0.4(-0.9 \\
0.1)\end{array}$ & 0.93 \\
\hline Bachelors & 60 & 60 & $\begin{array}{l}0.0 \\
(0.0 \\
0.0)\end{array}$ & $\begin{array}{l}3.6 \\
(0.5 \\
21.6)\end{array}$ & $\begin{array}{l}3.6 \\
(0.5 \\
21.6)\end{array}$ & $\begin{array}{l}3.6 \\
(0.5 ; \\
21.6)\end{array}$ & $\begin{array}{l}0.0(-0.4 \\
0.4)\end{array}$ & 1.15 \\
\hline Masters & 19 & 19 & $\begin{array}{l}0.0 \\
(0.0 \\
0.0)\end{array}$ & $\begin{array}{l}2.0 \\
(0.5 \\
7.6)\end{array}$ & $\begin{array}{l}3.9 \\
(1.5 \\
10.0)\end{array}$ & $\begin{array}{l}1.0 \\
(0.1 ; \\
6.7)\end{array}$ & $\begin{array}{l}-0.1(-0.3 \\
0.1)\end{array}$ & 1.05 \\
\hline
\end{tabular}




\begin{tabular}{|c|c|c|c|c|c|c|c|c|}
\hline Group & $\begin{array}{l}\text { Weighted } \\
N\end{array}$ & $\begin{array}{l}\text { Unweighted } \\
N\end{array}$ & $\begin{array}{l}-3 S D \\
(95 \% \\
C I)\end{array}$ & $\begin{array}{l}-2 S D \\
(95 \% \\
C I)\end{array}$ & $\begin{array}{l}+2 S D \\
(95 \% \\
C I)\end{array}$ & $\begin{array}{l}+3 S D \\
(95 \% \\
C I)\end{array}$ & $\begin{array}{l}\text { z-score } \\
\text { mean } \\
(95 \% \mathrm{Cl})\end{array}$ & $\begin{array}{l}Z- \\
\text { score } \\
\text { SD }\end{array}$ \\
\hline $\begin{array}{l}\text { No } \\
\text { Education }\end{array}$ & 58 & 58 & $\begin{array}{l}0.0 \\
(0.0 \\
0.0)\end{array}$ & $\begin{array}{l}1.7 \\
(0.2 \\
11.4)\end{array}$ & $\begin{array}{l}1.7 \\
(0.2 \\
11.4)\end{array}$ & $\begin{array}{l}1.7 \\
(0.2 \\
11.4)\end{array}$ & $\begin{array}{l}0.4(0.1 ; \\
0.7)\end{array}$ & 1.14 \\
\hline Primary & 78 & 78 & $\begin{array}{l}0.0 \\
(0.0 \\
0.0)\end{array}$ & $\begin{array}{l}0.0 \\
(0.0 \\
0.0)\end{array}$ & $\begin{array}{l}3.8 \\
(1.2 ; \\
11.3)\end{array}$ & $\begin{array}{l}0.0 \\
(0.0 \\
0.0)\end{array}$ & $\begin{array}{l}0.2(-0.1 \\
0.4)\end{array}$ & 1.02 \\
\hline Secondary & 19 & 19 & $\begin{array}{l}0.0 \\
(0.0 \\
0.0)\end{array}$ & $\begin{array}{l}0.0 \\
(0.0 \\
0.0)\end{array}$ & $\begin{array}{l}0.0 \\
(0.0 \\
0.0)\end{array}$ & $\begin{array}{l}0.0 \\
(0.0 \\
0.0)\end{array}$ & $\begin{array}{l}0.3(0.0 \\
0.7)\end{array}$ & 0.78 \\
\hline $\begin{array}{l}\text { Higher } \\
\text { Secondary }\end{array}$ & 9 & 9 & $\begin{array}{l}0.0 \\
(0.0 \\
0.0)\end{array}$ & $\begin{array}{l}11.1 \\
(1.5 \\
50.3)\end{array}$ & $\begin{array}{l}0.0 \\
(0.0 \\
0.0)\end{array}$ & $\begin{array}{l}0.0 \\
(0.0 \\
0.0)\end{array}$ & $\begin{array}{l}-0.4(-1.0 \\
0.3)\end{array}$ & 1.09 \\
\hline Bachelors & 33 & 33 & $\begin{array}{l}0.0 \\
(0.0 \\
0.0)\end{array}$ & $\begin{array}{l}0.0 \\
(0.0 \\
0.0)\end{array}$ & $\begin{array}{l}3.0 \\
(0.4 ; \\
18.8)\end{array}$ & $\begin{array}{l}0.0 \\
(0.0 \\
0.0)\end{array}$ & $\begin{array}{l}-0.1(-0.5 ; \\
0.2)\end{array}$ & 0.96 \\
\hline Masters & 102 & 102 & $\begin{array}{l}0.0 \\
(0.0 \\
0.0)\end{array}$ & $\begin{array}{l}2.9 \\
(0.9 \\
8.8)\end{array}$ & $\begin{array}{l}2.0 \\
(0.5 ; \\
7.6)\end{array}$ & $\begin{array}{l}2.0 \\
(0.5 ; \\
7.6)\end{array}$ & $\begin{array}{l}-0.2(-0.4 \\
0.0)\end{array}$ & 1.04 \\
\hline \multicolumn{9}{|c|}{ Child's Ethnicity: } \\
\hline Brahmin & 15 & 15 & $\begin{array}{l}0.0 \\
(0.0 \\
0.0)\end{array}$ & $\begin{array}{l}0.0 \\
(0.0 \\
0.0)\end{array}$ & $\begin{array}{l}0.0 \\
(0.0 \\
0.0)\end{array}$ & $\begin{array}{l}0.0 \\
(0.0 \\
0.0)\end{array}$ & $\begin{array}{l}-0.1(-0.4 \\
0.3)\end{array}$ & 0.79 \\
\hline Chhetri & 34 & 34 & $\begin{array}{l}0.0 \\
(0.0 \\
0.0)\end{array}$ & $\begin{array}{l}0.0 \\
(0.0 \\
0.0)\end{array}$ & $\begin{array}{l}2.9 \\
(0.4 ; \\
18.3)\end{array}$ & $\begin{array}{l}0.0 \\
(0.0 ; \\
0.0)\end{array}$ & $\begin{array}{l}0.1(-0.3 \\
0.4)\end{array}$ & 1.09 \\
\hline Dalit & 6 & 6 & $\begin{array}{l}0.0 \\
(0.0 \\
0.0)\end{array}$ & $\begin{array}{l}0.0 \\
(0.0 \\
0.0)\end{array}$ & $\begin{array}{l}0.0 \\
(0.0 \\
0.0)\end{array}$ & $\begin{array}{l}0.0 \\
(0.0 \\
0.0)\end{array}$ & $\begin{array}{l}-0.6(-1.3 \\
0.1)\end{array}$ & 0.91 \\
\hline Janajati & 82 & 82 & $\begin{array}{l}0.0 \\
(0.0 \\
0.0)\end{array}$ & $\begin{array}{l}0.0 \\
(0.0 \\
0.0)\end{array}$ & $\begin{array}{l}2.4 \\
(0.6 ; \\
9.3)\end{array}$ & $\begin{array}{l}1.2 \\
(0.2 ; \\
8.2)\end{array}$ & $\begin{array}{l}-0.1(-0.3 ; \\
0.1)\end{array}$ & 0.99 \\
\hline Newar & 166 & 166 & $\begin{array}{l}0.0 \\
(0.0 \\
0.0)\end{array}$ & $\begin{array}{l}3.0 \\
(1.3 \\
7.1)\end{array}$ & $\begin{array}{l}2.4 \\
(0.9 \\
6.3)\end{array}$ & $\begin{array}{l}1.2 \\
(0.3 \\
4.7)\end{array}$ & $\begin{array}{l}0.1(0.0 ; \\
0.3)\end{array}$ & 1.09 \\
\hline \multicolumn{9}{|c|}{ Birthweight Status } \\
\hline LBW & 30 & 30 & $\begin{array}{l}0.0 \\
(0.0 \\
0.0)\end{array}$ & $\begin{array}{l}0.0 \\
(0.0 \\
0.0)\end{array}$ & $\begin{array}{l}0.0 \\
(0.0 \\
0.0)\end{array}$ & $\begin{array}{l}0.0 \\
(0.0 ; \\
0.0)\end{array}$ & $\begin{array}{l}-0.3(-0.6 \\
0.1)\end{array}$ & 0.89 \\
\hline
\end{tabular}




\begin{tabular}{|c|c|c|c|c|c|c|c|c|}
\hline Group & $\begin{array}{l}\text { Weighted } \\
\boldsymbol{N}\end{array}$ & $\begin{array}{l}\text { Unweighted } \\
N\end{array}$ & $\begin{array}{l}-3 S D \\
(95 \% \\
C I)\end{array}$ & $\begin{array}{l}-2 S D \\
(95 \% \\
C I)\end{array}$ & $\begin{array}{l}+2 S D \\
(95 \% \\
C I)\end{array}$ & $\begin{array}{l}+3 S D \\
(95 \% \\
C I)\end{array}$ & $\begin{array}{l}\text { z-score } \\
\text { mean } \\
(95 \% \text { Cl) }\end{array}$ & $\begin{array}{l}z- \\
\text { score } \\
S D\end{array}$ \\
\hline NBW & 268 & 268 & $\begin{array}{l}0.0 \\
(0.0 \\
0.0)\end{array}$ & $\begin{array}{l}1.9 \\
(0.8 \\
4.4)\end{array}$ & $\begin{array}{l}2.6 \\
(1.2 ; \\
5.4)\end{array}$ & $\begin{array}{l}1.1 \\
(0.4 ; \\
3.4)\end{array}$ & $\begin{array}{l}0.1(-0.1 ; \\
0.2)\end{array}$ & 1.06 \\
\hline \multicolumn{9}{|c|}{ Delivery Type } \\
\hline $\begin{array}{l}\text { Cesarean } \\
\text { Section }\end{array}$ & 113 & 113 & $\begin{array}{l}0.0 \\
(0.0 \\
0.0)\end{array}$ & $\begin{array}{l}0.9 \\
(0.1 \\
6.1)\end{array}$ & $\begin{array}{l}2.7 \\
(0.9 \\
8.0)\end{array}$ & $\begin{array}{l}0.9 \\
(0.1 ; \\
6.1)\end{array}$ & $\begin{array}{l}0.2(0.1 \\
0.4)\end{array}$ & 0.95 \\
\hline Vaginal & 190 & 190 & $\begin{array}{l}0.0 \\
(0.0 \\
0.0)\end{array}$ & $\begin{array}{l}2.1 \\
(0.8 \\
5.5)\end{array}$ & $\begin{array}{l}2.1 \\
(0.8 \\
5.5)\end{array}$ & $\begin{array}{l}1.1 \\
(0.3 \\
4.1)\end{array}$ & $\begin{array}{l}-0.1(-0.2 ; \\
0.1)\end{array}$ & 1.09 \\
\hline
\end{tabular}

\section{Discussion}

Our study unearthed many important findings, some expected and some unexpected, on IYCF practices, consumption of nutrient-poor packaged foods and child malnutrition in the urban and peri-urban context of Nepal. Due to paucity of recent data, the comparison on the IYCF indicators are mainly made with the urban disaggregation of the last two national surveys which are both from 2016.

We found mixed results on the indicators related to breastfeeding. Our study did not collect data on exclusive breastfeeding. But the collected breastfeeding indicators are poorer than those reported by the 'urban' disaggregation of the national surveys. Any breastfeeding is universal and is still very high until at least around one year of age. However, early initiation of breastfeeding within the recommended one hour of birth and the practice of bottle feeding were not optimal. For instance, the NMNSS 2016 which reported EIBF among 6-23 months had found it to be $70.1 \%$ while our finding showed $47.9 \%$ only. Similarly, the same survey reported the proportion of urban children using bottle with a nipple was $20.7 \%$ while this study showed $48.5 \%$. There could be two explanations for such differences - either those indicators have progressively worsened over the last few years or the large scale survey combining all urban areas had masked the situation in specific and densely populated area like Bhaktapur. Breastfeeding is well established as vital for child survival and optimal child development with a host of benefits in any context for short and long term and thus ought to be upheld. $(12,13)$ Our findings prompt towards paying specific attention to accelerate the protection, promotion and support of breastfeeding in Nepal's urban context.

Our study found comparatively better status of the MDD (66\%), in comparison to that reported by both NMNSS 2016 (49.9\%) and NDHS 2016 (48\%) for the urban children of the same age group. On the other hand, MMF at $79.1 \%$ was slightly lower than that reported by NMNSS $2016(84.3 \%)$ but higher than that reported by the NDHS 2016 (70.8\%). Overall, the young urban children from our study had better MAD (54.4\%) compared to both NDHS 2016 (36.4\%) and NMNSS 2016 (39.1\%). Nevertheless, our findings 
indicate some positive trends on the IYCF practices while also pointing out that much remains to be done to improve diet of the youngest age groups in urban areas of Nepal.

The findings we have unearthed on consumption of nutrient-poor packaged foods add troubling dimensions to the prevailing feeding practices among urban Nepali children. These are also not captured through the WHO recommended standard IYCF indicators by national surveys. The fact that our study documented two-thirds (63\%) of the small children to be consuming nutrient-poor packaged foods ought to warrant attention. Biscuits were the most commonly given packaged food which were often displacing

the proper meals. The NMNSS 2016 had documented almost universal consumption (95.6\%) of biscuits and cookies by Nepali households which might make it an easy and appealing option to give to the children too. We also found that more than a third were given tea and often with the biscuits. The NMNSS 2016 had also reported similar proportion of $(31.8 \%)$ of tea consumption among urban children aged 623 months. This has dual consequences for poor child nutrition - on one hand they are getting nutrient poor commercial snack foods that also tend to replace proper meals. On the other hand the inhibitory compounds present can interfere with absorption of critical nutrient like iron among an already vulnerable population affecting their mental and physical developments.(14) Additional worrying finding from our study is that mostly mothers themselves (64.1\%), the primary caregivers of the majority of the children, were giving these foods to their babies. A recent study had found that most families live within a five minutes-walk to a store selling unhealthy processed foods.(15)A 2016 study conducted in Kathmandu valley reported that $85.4 \%$ of mothers had seen promotion of nutrient-poor foods for children and $74.1 \%$ of children aged 6-23 months had consumed those foods.(16) A recent study also conducted in Kathmandu valley had found that $48.5 \%$ of caregivers gave convenience as a major reason for giving such foods.(17) In addition to convenience, other reasons could range from lack of knowledge about poor nutrient composition of those foods, exposure to constant advertisements, easy availability or combinations of such factors. Early life taste and food preferences are considered important to influence later food choices and health, foods high in sugar, salt and fat during complementary feeding period increases predisposition towards such foods later in life too.(18) The underlying drivers for this have to be comprehensively understood and need to be holistically addressed.

Child undernutrition prevalence documented by our study was significantly lower in comparison to the urban disaggregation of the aforementioned national surveys. As expected, children born with normal birth weight had lower rates of stunting and underweight. However, our findings on child wasting was perplexing. Of those children who were wasted, all were reported to have been born normal weight. Another significant finding is that amongst this sample of young children, the prevalence of overweight and obesity is higher than wasting which is something to be wary of. Whilst our study did not look at associations, one possible reason could be that the association with increasing consumption of high calorie but nutrient poor unhealthy foods. A recent study had explored and showed an association of nutrient-poor packaged foods consumption with inadequate nutrient intakes and poor child growth among urban children in urban Kathmandu.(19) Such association needs to be explored with regards to early childhood overweight and obesity too in this setting, also concurred by 
finding of a related systematic review. (20) This is especially necessary in view of that poor quality of diet in early childhood have been linked to adiposity in later childhood. $(21,22)$ Furthermore, excessive weight gain in early childhood predisposes children to obesity and non-communicable diseases later on in life too.(23)

Our study had certain limitations. Due to resource constraints, a larger sample size was not possible, thus some of the results have wide confidence intervals. There is a chance that the higher MDD documented by our study could be due respondents' social desirability bias. Also, our study was not designed to be able to conduct logistics regression analysis which could have helped to explain the association of some of our findings.

This study adds to the evidence that practices related to breastfeeding and diets of young children in urban areas of Nepal are not optimal with only around half meeting the minimum standards recommended by the WHO. There are also apparent shifts towards the consumption of commercially produced nutrient-poor foods and indications of rising overweight and obesity. Targeted urban nutrition responses have not yet fallen within the purview of the government or development agencies as the prevailing issues in rural areas still get the focus and the priority. The rapid pace of urbanization taking place in Nepal should not be forgotten. Global calls have been made for double-duty actions to holistically address undernutrition, overweight, obesity and diet-related non-communicable diseases in order to end malnutrition in all its forms.(24) Therefore, there is a pressing need to wake up to these unfolding malnutrition realities in urban areas of Nepal and carve out necessary urban nutrition policies and programmatic interventions accordingly.

Multi-pronged approaches are required that bring systemic changes to curtail harmful advertising and marketing of breast milk substitutes and unhealthy commercial foods as well as explore the potential role of urban and/or peri-urban agriculture in shaping diets and nutrition. $(25,26)$ At the same time, nutrition social behaviour change communication ought to be introduced and accelerated through innovative ways and utilising non-traditional platforms like hospitals, day-care centers and preschools to widen the reach and ensure that no child is left behind in the spirit of Sustainable Development Goals.

Conclusion: The rising adverse nutrition issues among young children in the urban and peri-urban Nepali context can still be nipped in the bud before they get too pervasive. We end with an urgent call to policy makers, programme planners and researchers to pay attention to the rapidly changing dietary patterns among young children and the associated child malnutrition emerging in the urban areas of Nepal.

More studies should be conducted with larger sample sizes in multiple settings including urban hospitals as well as urban and peri-urban communities. Future studies should also look at better understanding the

underlying determinants as well as associations and correlations with the consumption of nutrient-poor packaged food among young children. Such unhealthy foods consumption ought to be captured by national surveys too, especially by the periodic national surveys like the NDHS. Kathmandu valley, being the most densely populated urban area of Nepal, should be presented by national surveys as a special 
disaggregation as combining it with other urban areas might have diluted and skewed the actual situation.

\section{List Of Abbreviations}

WHO: World Health Organization

IYCF: Infant and young child feeding

MDD: Minimum Dietary Diversity

MMF: Minimum Meal Frequency

MAD: Minimum Acceptable Diet

NDHS: Nepal Demographic Health Survey

$\mathrm{MOH}$ : Ministry of Health

NMNSS: Nepal Micronutrient Status Survey

UNICEF: United Nations International Children's Education Fund

ARCH: Assessment in Child Feeding and Health

\section{Declarations}

Ethics approval and consent to participate: Ethical approval was obtained from Nepal Health Research Council (NHRC reference number 718 - 2019) to conduct this research. All the eligible children and their parents were approached and a written informed consent was obtained from patents before enrolling them in the study.

Consent for publication: Not applicable

Availability of data and materials: De-identified data and related material of this study are available upon reasonable request from the corresponding author.

Competing interests: None.

Funding: No external funding was received for this work

Authors' contributions: S.U. conceptualized the study, conducted data analysis and wrote the paper; A.K. assisted in designing the study, supported pre-testing of the questionnaires, conducted data collection and assisted in quality monitoring; A. P. supported pre-testing of the questionnaires and conducted data 
collection; D.S. supported the study process by reviewing the study design, quality monitoring and reviewing the draft manuscript. All authors have approved the final manuscript for publication.

\section{Acknowledgements:}

We are grateful to the management of Siddhi Memorial Hospital and immunization unit for their kind support and determination during this research and would like to thank all the child and parents who participated in this study.

\section{References}

1. Ministry of Health, Nepal;New Era;and ICF. 2017, Nepal Demographic and Health Survey 2016. Kathmandu, Nepal:Ministry of Health, Nepal.

2. De Onis M, Borghi E, Arimond M, Webb P, Croft T, Saha K, et al. Prevalence thresholds for wasting, overweight and stunting in children under 5 years. Public Health Nutrition. 2019;22(1):175-9.

3. Victora CG, Adair L, Fall C, Hallal PC, Martorell R, Richter L, et al. Maternal and child undernutrition: consequences for adult health and human capital. The Lancet. 2008;371(9609):340-57.

4. Popkin BM. Nutritional Patterns and Transitions. Population and Development Review. 1993;19(1).

5. Bakrania S. Urbanisation and urban growth in Nepal (GSDRC Helpdesk Research Report 1294). In: Birmingham UG, editor. GSDRC Helpdesk Research Report: University of Birmingham; 2015.

6. Black RE, Victora CG, Walker SP, Bhutta ZA, Christian P, de Onis M, et al. Maternal and child undernutrition and overweight in low-income and middle-income countries. The Lancet. 2013;382(9890):427-51.

7. Jaacks LM, Kavle J, Perry A, Nyaku A. Programming maternal and child overweight and obesity in the context of undernutrition: current evidence and key considerations for low- and middle-income countries. Public Health Nutr. 2017;20(7):1286-96.

8. Ministry of Health and Population, Nepal; New Era; UNICEF; EU; USAID; and CDC.2018. Nepal National Micronutrient Status Survey Report 2016. Kathmandu, Nepal:Ministry of Health and Population, Nepal.

9. Angdembe MR, Dulal BP, Bhattarai K, Karn S. Trends and predictors of inequality in childhood stunting in Nepal from 1996 to 2016. Int J Equity Health. 2019;18(1):42.

10. UNICEF. State of World's Children 2019, Children, food and nutrition: Growing well in a changing world. In: UNICEF, editor. Newyork2019.

11. Pries AM, Sharma N, Upadhyay A, Rehman AM, Filteau S, Ferguson EL. Energy intake from unhealthy snack food/beverage among 12-23-month-old children in urban Nepal. Matern Child Nutr. 2019;15 Suppl 4:e12775.

12. Victora CG, BahI R, Barros AJD, França GVA, Horton S, Krasevec J, et al. Breastfeeding in the 21st century: epidemiology, mechanisms, and lifelong effect. The Lancet. 2016;387(10017):475-90. 
13. Rollins NC, Bhandari N, Hajeebhoy N, Horton S, Lutter CK, Martines JC, et al. Why invest, and what it will take to improve breastfeeding practices? The Lancet. 2016;387(10017):491-504.

14. EHM Temme, PGA Van Hoydonck.Tea consumption and iron status. European Journal of Clinical Nutrition. 2002;56;379-386. DOI: 10.1038/sj/ejcn/1601309

15. Tsang C, Sokal-Gutierrez K, Patel P, Lewis B, Huang D, Ronsin K, et al. Early Childhood Oral Health and Nutrition in Urban and Rural Nepal. Int J Environ Res Public Health. 2019;16(14).

16. Pries AM, Huffman SL, Adhikary I, Upreti SR, Dhungel S, Champeny M, et al. High consumption of commercial food products among children less than 24 months of age and product promotion in Kathmandu Valley, Nepal. Matern Child Nutr. 2016;12 Suppl 2:22-37.

17. Sharma N, Ferguson EL, Upadhyay A, Zehner E, Filteau S, Pries AM. Perceptions of commercial snack food and beverages for infant and young child feeding: A mixed-methods study among caregivers in Kathmandu Valley, Nepal. Matern Child Nutr. 2019;15 Suppl 4:e12711.

18. De Cosmi V, Scaglioni S, Agostoni C. Early Taste Experiences and Later Food Choices. Nutrients. 2017;9(2).

19. Pries AM, Rehman AM, Filteau S, Sharma N, Upadhyay A, Ferguson EL. Unhealthy Snack Food and Beverage Consumption Is Associated with Lower Dietary Adequacy and Length-for-Age z-Scores among 12-23-Month-Olds in Kathmandu Valley, Nepal. J Nutr. 2019;149(10):1843-51.

20. Pries AM, Filteau S, Ferguson EL. Snack food and beverage consumption and young child nutrition in low- and middle-income countries: A systematic review. Matern Child Nutr. 2019;15 Suppl 4:e12729.

21. Okubo H, Crozier SR, Harvey NC, Godfrey KM, Inskip HM, Cooper C, et al. Diet quality across early childhood and adiposity at 6 years: the Southampton Women's Survey. Int J Obes (Lond). 2015;39(10):1456-62.

22. Braun JM, Kalkwarf HJ, Papandonatos GD, Chen A, Lanphear BP. Patterns of early life body mass index and childhood overweight and obesity status at eight years of age. BMC Pediatr. 2018;18(1):161.

23. Richard J. Munthali JK, Zané Lombard, and Shane A. Norris. Early Life Growth Predictors of Childhood Adiposity Trajectories and Future Risk for Obesity: Birth to Twenty Cohort. Childhood obesity. 2017;13(5):384-91.

24. Hawkes C, Ruel MT, Salm L, Sinclair B, Branca F. Double-duty actions: seizing programme and policy opportunities to address malnutrition in all its forms. The Lancet. 2020;395(10218):142-55.

25. Corrina Moucheraud RKC, Sigrun Henjum, Tor A Strand, Manjeswori Ulak, Wafaie W Fawzi, Lindsey M Locks, Andrew L Thorne-Lyman, Patrick Webb. Engagement in Agriculture Protects Against Food Insecurity and Malnutrition in Peri-Urban Nepal. Current Developments in Nutrition. 2019;3:nzy078.

26. Clark H, Coll-Seck AM, Banerjee A, Peterson S, Dalglish SL, Ameratunga S, et al. A future for the world's children? A WHO-UNICEF-Lancet Commission. The Lancet. 2020;395(10224):605-58. 